\title{
合成 2,5-二芳基噻唑衍生物的新方法
}

\author{
曾鸿运 $a$ 张军干 $a$ 洪 伟*, $a, b$ \\ ( ${ }^{a}$ 北方民族大学化学与化学工程学院 银川 750021) \\ $\left({ }^{b}\right.$ 北方民族大学国家民委化工技术基础重点实验室 银川 750021)
}

\begin{abstract}
摘要 噻唑环作为一类重要的五元芳香杂环, 其衍生物具有多种生物活性, 被广泛应用在医药方面. 以价廉易得的不 同取代苯甲酸为原料，经酰化反应、硫代反应、环合反应和 Heck 反应合成了 2,5-二芳基噻唑衍生物. 重点在于优化 Heck 反应的条件, 进行可能反应机理的探讨. 该方法具有反应条件温和、操作简单及底物的普适性良好等优点, 为 2,5-二芳 基取代噻唑类化合物的合成提供了新的方向.
\end{abstract}

关键词 2,5-二芳基噻唑; Heck 反应; 有机合成

\section{New Method for the Synthesis of 2,5-Diaryl Substituted Thiazoles}

\author{
Zeng, Hongyun $^{a} \quad$ Zhang, Jun'gan ${ }^{a} \quad$ Hong, Wei*,a,b \\ ( ${ }^{a}$ School of Chemistry and Chemical Engineering, North Minzu University, Yinchuan 750021) \\ $\left({ }^{b}\right.$ Key Laboratory of Chemical Engineering and Technology, State Ethnic Affairs Commission, North Minzu \\ University, Yinchuan 750021)
}

\begin{abstract}
Thiazole ring is an important five-membered aromatic heterocyclic ring, and its derivatives have various biological activities and are widely used in medicine. The synthesis of 2,5-diarylthiazole derivatives by acylation, thiolation, cyclization and Heck reaction using inexpensive and readily available substituted benzoic acid as raw materials was developed. The key point was to optimize the Heck reaction conditions and explore the possible reaction mechanism. The method has mild reaction conditions, simple operation, and good substrate universality, which provides a new direction for the synthesis of 2,5-diaryl substituted thiazoles.
\end{abstract}

Keywords 2,5-diarylthiazole; Heck reaction; organic synthesis

噻唑环是一类重要的含有氮和硫原子的五元芳香 杂环, 具有富电子性, 可以通过氢键、配位键、范德华 力和静电等多种非共价键与生物体内的多种酶、蛋白 质、核酸及其他活性位点发生作用. 噻唑类化合物显示 出较好的生物活性, 如抗细菌 ${ }^{[1-4]}$ 、抗真菌 ${ }^{[5-8]}$ 、抗结 核 ${ }^{[9-11]}$ 、抗病毒 ${ }^{[12-14]}$ 、抗肿瘤 ${ }^{[15-18]}$ 和抗炎 ${ }^{[19-21]}$ 等. 因此, 近年来噻唑类化合物在医药方面的研究和应用较为广 泛, 众多噻唑类药物已经成为临床一线药物, 比如抗细

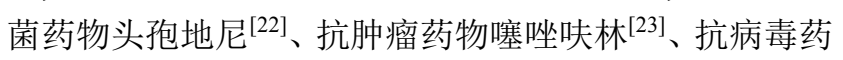
物利托那韦 ${ }^{[24]}$ 、消炎镇痛药物美洛昔康 ${ }^{[25]}$ 和抗寄生虫药 物尼里达唑 ${ }^{[26]}$ 等. 此外, 还有很多从天然产物中分离出 来的含噻唑环的活性分子, 比如从 Scleritoderma 海绵中 分离得到活性环肽 Scleritodermin $\mathrm{A}^{[27-28]}$ 、从菲律宾南部
的 Lissoclinum bistratum 属海鞘中分离得到海洋环肽 Bistratamides ${ }^{[29}$ 和从海洋蓝藻菌鞘丝藻属中分离得到环 肽 Obyanamide ${ }^{[30]}$ 等.

目前, 关于 2,5-二芳基噻唑-4-甲酸乙酯衍生物合成 方法的报道较少. 在 2002 年, Hodgetts 等 ${ }^{[31]}$ 报道了以 2氨基噻唑-4-甲酸乙酯为原料, 经溴取代反应得到 2-澳 噻唑-4-甲酸乙酯, 再经过氯取代反应得到 2-澳-5-氯噻 唑-4-甲酸乙酯, 然后先后在 C(2)位和 C(5)位经 Suzuki 反应以较高收率得到 2,5-二苯基噻唑-4-甲酸乙酯衍生 物. 该方法存在的问题在于 2 位的碳溴键发生 Suzuki 反 应时, 5 位的碳氯键可能也会参与, 从而影响反应的选 择性. 另外, 此方法合成步骤较长, 后处理纯化次数较 多. 近年来对噻唑环的 $\mathrm{C}(5)$ 位引入苯基的研究较为广

\footnotetext{
* Corresponding author. E-mail: hongwei@nmu.edu.cn

Received April 13, 2020; revised May 22, 2020; published online May 29, 2020.

Project supported by the National Natural Science Foundation of China (Nos. 81660588, 81960623) and the National Ethnic Affairs Commission Young Talent Training Program.

国家自然科学基金(Nos. 81660588, 81960623)和国家民委中青年英才培养计划资助项目.
} 
泛, 通常以 $\mathrm{C}(2)$ 位为氨基或者是烷基取代的噻唑通过 Heck 反应得到偶联产物, 而没有以 $\mathrm{C}(2)$ 位为苯基取代 的噻唑作为反应底物的报道. 比如在 2007 年, Priego 等 ${ }^{[32]}$ 报道了以 2-(叔丁氧基羰基氨基)噻唑-4-甲酸乙酯 和取代碘苯为原料, 碳酸铯为碱, 醋酸钯为催化剂, 2二环己膦基-2-( $N, N$-二甲胺)-联苯为配体, $N, N$-二甲基甲 酰胺(DMF) 为溶剂, 以较高收率得到 C(5)位芳基化产物. 可以看出这类反应的关键条件在于配体、溶剂和碱的选 择. 在 2012 年, Beladhria 等 ${ }^{[33]}$ 报道了以 2-甲基噻唑-4甲酸乙酯和 2-氯-4-氨基碘苯为原料, 醋酸钾为碱, 醋酸 钯为催化剂, $N, N$-二甲基乙酰胺(DMAc)为溶剂, 在 C(5) 位直接进行芳基化. 从已经报道的方法看, 在 $\mathrm{C}(5)$ 位进 行 Heck 反应时碳酸铯和醋酸钾为常用碱, $N$-甲基吡咯 烷酮 $(\mathrm{NMP})^{[34]}$ 、DMAc 和 DMF 等高沸点溶剂为较常用 的溶剂, 配体的选择多为带有膦基的配体.

综合上述方法, 我们介绍一种合成 2,5-二芳基噻 唑-4-甲酸乙酯衍生物的新方法(Scheme 1): 以取代苯甲 酸 $1 \mathrm{a} \sim 1 \mathrm{~g}$ 为原料经酰化反应和硫代反应 ${ }^{[35]}$ 得到取代的 硫代酰胺 $2 \mathrm{a} \sim 2 \mathrm{~g}$, 再与溴代丙酮酸乙酯发生环合反 应 ${ }^{[36-38]}$ 得到 2-苯基噻唑-4-甲酸乙酯 $3 \mathbf{a} \sim \mathbf{3 g}$, 最后经过 Heck 反应得到目标化合物 $\mathbf{5 a} \sim \mathbf{5 1}$. 其关键步骤为以 2 苯基噻唑-4-甲酸乙酯衍生物 $3 \mathbf{a} \sim 3 \mathbf{g}$ 为原料, 在 $\mathrm{C}(5)$ 位 直接芳基化. 该方法的优点在于: 首先在形成噻唑环这 一步反应, 噻坐 $\mathrm{C}(2)$ 位直接引入苯基, 避免上述两次 Suzuki 反应带来的选择性问题; 其次在 C(5)位经 Heck 反应引入苯基, 相比上述的氯代反应和 Suzuki 反应, 减 少反应步骤, 提高反应路线的效率. 另外, 此类化合物 $\mathrm{C}(4)$ 位为酯基, 后续可对其改造并合成一些具有一定生 物活性的化合物.<smiles>O=C(O)c1[R1]ccc1</smiles>

$1 \mathrm{a} \sim 1 \mathrm{~g}$<smiles>CCOC(=O)CBr</smiles>

EtOH

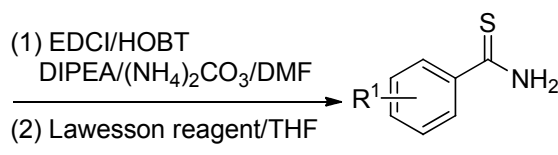

$2 \mathrm{a} \sim 2 \mathrm{~g}$<smiles></smiles>

$3 \mathbf{a} \sim 3 \mathbf{g}$

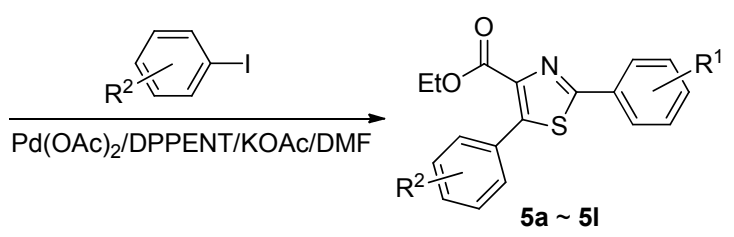

图式 12 2,5-二芳基噻唑-4-甲酸乙酯衍生物的合成 Scheme 1 Synthesis of 2,5-diarylthiazole-4-ethyl formate derivatives

\section{1 结果与讨论}

\subsection{2-苯基噻唑-4-甲酸乙酯与 4-甲氧基碘苯反应条件} 的确定

2,5-二芳基噻唑-4-甲酸乙酯类化合物的合成是由可 商业购买的取代苯甲酸为原料经酰化反应、硫代反应、 环合反应和 Heck 反应得到. 由于中间体 2-苯基噻唑-4甲酸乙酯与碘苯发生的 Heck 反应是此路线的关键步骤, 因此为了能高效地得到目标化合物, 我们以 2-苯基噻 唑-4-甲酸乙酯(3a)和 4-甲氧基碘苯(4a)为底物建立模板 反应，通过考察配体的种类及用量、碱的种类、溶剂的 种类及用量、时间和温度等因素，确定其最佳反应条件， 结果如表 1 所示. 首先, 为了确定适合的配体, 分别尝 试三种结构不同的配体 $[1,4-$ 双(二苯基膦)丁烷(DPPH)、 1.5-双(二苯基膦)戊烷(DPPENT)和 1,6-双(二苯基膦)丁 烷 (DPPB)](图 1), 以醋酸钯为催化剂, 醋酸钾为碱, NMP 为溶剂, 反应温度为 $140{ }^{\circ} \mathrm{C}$ 时, 发现 DPPENT 为 配体时, 能以较好的产率(35\%)得到产物 5a. 因此，选 择 DPPENT 为最佳配体(表 1, Entries 1 3). 然后, 考察 了碱对该反应的影响，在相同的反应条件下，对比碳酸 铯和碳酸钾的反应效果, 发现这两种碱均未能得到产物 5a. 因此, 确定乙酸钾为碱(表 1 , Entries 2, 4, 5). 在确定 最佳配体与碱之后，考察不同溶剂对该反应的影响，发 现 二甲基乙酰胺为溶剂时反应收率与 NMP 相当，但是 $N, N$-二甲基甲酰胺为溶剂时反应收率有所提高, 达到了 45\%(表 1, Entries 2, 6, 7).

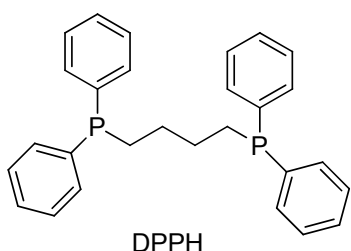

$\mathrm{DPPH}$

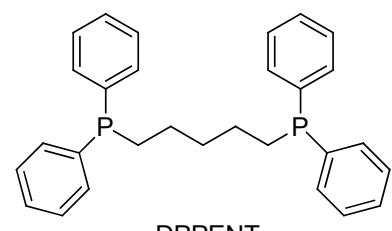

DPPENT<smiles>O=P(O)([Pb])CCCCCP(c1ccccc1)c1ccccc1</smiles>

图 1 配体 DPPH、DPPENT 和 DPPB 的结构式

Figure 1 Structures of ligands DPPH, DPPENT and DPPB

接下来, 我们探索反应浓度、时间、温度以及配体 用量对 2-苯基噻唑-4-甲酸乙酯与 4-甲氧基碘苯反应的 影响. 当改变溶剂 DMF 的用量为 $2 \mathrm{~mL}$, 增加反应物的 浓度后，反应收率为 $31 \%$ ，表明增加反应物浓度反而使 收率降低(表 2, Entries 7,8). 当改变反应时间, 将时间 缩短至 $12 \mathrm{~h}$, 反应收率为 $38 \%$, 说明缩短反应时间使收 率降低(表 2, Entries 7,9). 随后,降低反应温度至 120 
表 1 配体、碱和溶剂对 2-苯基塞唑-4-甲酸乙酯与 4-甲氧基 碘苯反应的影响 ${ }^{a}$

Table 1 Effects of ligand, base and solvent on reaction of 2phenylthiazole-4-ethylformate with 4-methoxy-iodobenzene
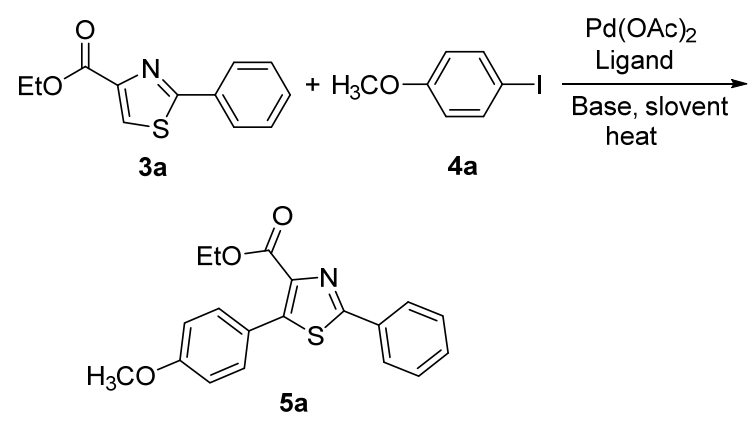

\begin{tabular}{ccccc}
\hline Entry & Ligand & Base & Solvent & Yield $^{b} \%$ \\
\hline 1 & DPPH & KOAc & NMP & 27 \\
2 & DPPENT & KOAc & NMP & 35 \\
3 & DPPB & KOAc & NMP & 24 \\
4 & DPPENT & $\mathrm{CS}_{2} \mathrm{CO}_{3}$ & NMP & - \\
5 & DPPENT & $\mathrm{K}_{2} \mathrm{CO}_{3}$ & NMP & - \\
6 & DPPENT & KOAc & DMA & 31 \\
7 & DPPENT & KOAc & DMF & 45 \\
\hline
\end{tabular}

${ }^{a}$ Reaction conditions: 3a (1 mmol), 4-methoxy-iodobenzene (3 mmol), base (3 $\mathrm{mmol})$, ligand $(0.01 \mathrm{mmol}), \mathrm{Pd}(\mathrm{OAc})_{2}(0.05 \mathrm{mmol})$ in solvent $(4 \mathrm{~mL})$ at $140{ }^{\circ} \mathrm{C}, 24 \mathrm{~h} ;{ }^{b}$ Isolated yield.

表 2 反应浓度、时间、温度和配体用量对 2-苯基噻唑-4 甲酸 乙酯与 4-甲氧基碘苯反应的影响 ${ }^{a}$

Table 2 Concentration, time, temperature and ligand equivalent effects on reaction of 2-phenylthiazole-4-ethylformate with 4-methoxy-iodobenzene

\begin{tabular}{cccccc}
\hline Entry & Ligand & Solvent & Temp. $/{ }^{\circ} \mathrm{C}$ & Time $/ \mathrm{h}$ & Yield $^{b} \%$ \\
\hline 7 & DPPENT & DMF & 140 & 24 & 45 \\
8 & DPPENT & DMF $^{c}$ & 140 & 24 & 31 \\
9 & DPPENT & DMF & 140 & 12 & 38 \\
10 & DPPENT $^{d}$ & DMF & 120 & 24 & 48 \\
11 & DPPENT $^{d}$ & DMF & 120 & 24 & 45 \\
12 & DPPENT $^{e}$ & DMF & 120 & 24 & 31
\end{tabular}

${ }^{a}$ Reaction conditions: 3a (1 mmol), 4-methoxy-iodobenzene (3 mmol), KOAc (3 mmol), DPPENT (0.01 mmol), Pd(OAc) $2(0.05 \mathrm{mmol})$ in DMF $(4 \mathrm{~mL})$ at $140{ }^{\circ} \mathrm{C}, 24 \mathrm{~h} ;{ }^{b}$ Isolated yield; ${ }^{c}$ DMF $(2 \mathrm{~mL}) ;{ }^{d}$ DPPENT (0.02 mmol); ${ }^{e}$ DPPENT (0.04 mmol).

${ }^{\circ} \mathrm{C}$, 反应时间仍然保持 $24 \mathrm{~h}$, 收率为 $48 \%$, 但在考虑实 验的操作性, 选择以温度 $120{ }^{\circ} \mathrm{C}$ 为反应温度更佳(表 2 , Entries 7, 10). 最后, 当增加配体的物质的量分别至 0.02 和 $0.04 \mathrm{mmol}$, 反应收率相应为 $45 \%$ 与 $31 \%$, 随着配体 物质的量比的增加, 反应收率反而降低(表 2, Entries 7, 11，12). 最终确定 2-苯基噻唑-4-甲酸乙酯(3a)和 4-甲氧 基碘苯(4a)的模板反应条件为: 2-苯基噻坐-4-甲酸乙酯 (1 mmol)、4-甲氧基碘苯 $(3 \mathrm{mmol}) 、$ 醋酸钯 $(0.05 \mathrm{mmol})$ 、 1.5 -双 (二苯基膦)戊烷 $(0.01 \mathrm{mmol}$ )、碳酸钾 $(3 \mathrm{mmol})$, 溶 剂为 $N, N$-二甲基甲酰胺 $(4 \mathrm{~mL})$, 温度为 $120{ }^{\circ} \mathrm{C}$, 时间为 $24 \mathrm{~h}$.

\subsection{2,5-二芳基噻唑-4-甲酸乙酯衍生物的合成}

在确定最佳反应条件之后, 探究了一系列不同取代 基对该反应的影响, 反应结果如表 3 所示. 主要考察了 2,5-二芳基噻唑-4-甲酸乙酯的苯基上取代基 $\mathrm{R}^{1}$ 和 $\mathrm{R}^{2}$ 对 该反应的影响. 首先, 选择 $\mathrm{R}^{2}$ 为供电子基团的 4-甲氧 基, $\mathrm{R}^{1}$ 为不同取代基团(如氢原子、甲基、甲氧基、三氟 甲氧基、氟和氯)时对此反应的影响. 以 4-甲氧基碘苯 (4a)与不同取代的 2-苯基噻唑-4-甲酸乙酯 $3 \mathrm{a} \sim 3 \mathrm{~g}$ 为原 料, 在此反应条件下得到目标化合物 $\mathbf{5 a} \sim \mathbf{5 g}$. 当 $\mathrm{R}^{1}$ 为 无取代基时，此反应的收率为 48\%(表 3, Entry 1); $R^{1}$ 为 4-甲基、4-甲氧基、3-甲氧基、4-三氟甲氧基和 4-氟时，反 应的收率明显提高至 50\% 54\%(表 3, Entries 2 6); $\mathrm{R}^{1}$ 为 4-氯时, 此反应收率明显降低至 38\%(表 3, Entry 7). 然后选择 $\mathrm{R}^{2}$ 为吸电子基团的 4-硝基, $\mathrm{R}^{1}$ 为不同取代基团 (如氢原子、甲氧基和氟)时对此反应的影响. 以 4-硝基 碘苯(4b)分别与不同取代的 2-苯基噻唑-4-甲酸乙酯(3a、 3c、3f) 为原料, 在此反应条件下得到目标化合物 $\mathbf{5 h} \sim \mathbf{5 j}$, 收率为 $51 \% \sim 54 \%$ (表 3, Entries 8 10). 最后, 选择 $\mathrm{R}^{1}$ 为 4-甲氧基的 $3 \mathrm{c}$ 分别与 $\mathrm{R}^{2}$ 为供电子基团的 4-吗啉基和 3-甲氧基碘苯 $(4 \mathrm{c}$ 和 $4 d)$ 为原料, 得到目标化合物 $5 k$ 和 51, 收率均为 52\%(表 3, Entries 11, 12). 所有目标化合物 5a $\sim 51$ 的结构均经过 ${ }^{1} \mathrm{H}$ NMR, ${ }^{13} \mathrm{C}$ NMR 和 HRMS 的表 征.

\section{3 反应机理探讨}

通过实验结果分析, 我们推测出以 2-苯基噻唑-4甲酸乙酯(3a)和 4 -甲氧基碘苯(4a)为反应底物, 生成目 标产物 5a 可能的反应机理(Scheme 2). 首先, 醋酸钯和 1,5 -双 (二苯基膦)戊烷络合被还原为零价钯, 零价钯通 过氧化加成反应插入到 $\mathbf{4 a}$ 中的 $\mathrm{C}-\mathrm{I}$ 键间被氧化为二价 钯, 形成中间体 $\mathbf{A}$; 由于原料 $\mathbf{3 a}$ 的 $\mathrm{C}(5)-\mathrm{C}(4)$ 与酯基刚 好形成 $\alpha, \beta$-不饱和酯的结构, $\mathrm{C}(5)$ 位的电子云密度低, 可以被中间体 $\mathbf{A}$ 中与二价钯相邻的碳进攻, 同时双键打 开, C(4)进攻二价钯形成加成产物 $\mathbf{B}$; 然后加成产物 $\mathbf{B}$ 经过 $\beta$ 消除得到目标产物 $\mathbf{5 a}$; 最后二价钯通过醋酸钾的 还原消除作用, 还原为零价钯.

\section{2 结论}

提出了一种简便合成 2,5-二芳基噻唑-4-甲酸乙酯 衍生物的新方法. 该方法中, 首先使用取代苯甲酸经酰 化反应和硫代反应得到硫代酰胺化合物, 再经环合反应 得到 2-苯基噻唑-4-甲酸乙酯衍生物, 实现在噻唑环 C2 位引入苯基, 最后经 Heck 反应直接在 $\mathrm{C} 5$ 位引入苯基, 从而得到 2,5-二芳基噻唑-4-甲酸乙酯衍生物 5a $\sim 5$ I. 该 方法的反应条件温和, 操作简单, 纯化步骤较少, 总收 率较高. 
表 32 2,5-二芳基噻唑衍生物 $\mathbf{5 a} \sim \mathbf{5 1}$ 的合成 ${ }^{a}$

Table 3 Synthesis of 2,5-diarylthiazole-4-ethyl formate derivatives $\mathbf{5 a} \sim \mathbf{5 l}$<smiles>[R]c1ccc(-c2nc(C(=O)OCC)c(-c3cccc([R])c3)s2)cc1</smiles>

\begin{tabular}{|c|c|c|c|c|}
\hline Entry & $\mathrm{R}^{1}(\mathbf{3})$ & $\mathrm{R}^{2}(4)$ & Product & Yield $^{b} / \%$ \\
\hline 1 & 4-H (3a) & $4-\mathrm{OCH}_{3}(\mathbf{4 a})$ & $5 a$ & 48 \\
\hline 2 & $4-\mathrm{CH}_{3}(\mathbf{3 b})$ & $4-\mathrm{OCH}_{3}(\mathbf{4 a})$ & $5 b$ & 54 \\
\hline 3 & $4-\mathrm{OCH}_{3}(3 \mathbf{c})$ & $4-\mathrm{OCH}_{3}(\mathbf{4 a})$ & $5 c$ & 50 \\
\hline 4 & $3-\mathrm{OCH}_{3}(\mathbf{3 d})$ & $4-\mathrm{OCH}_{3}(\mathbf{4 a})$ & $5 d$ & 50 \\
\hline 5 & $4-\mathrm{OCF}_{3}(\mathbf{3 e})$ & $4-\mathrm{OCH}_{3}(\mathbf{4 a})$ & $5 e$ & 55 \\
\hline 6 & $4-F(3 f)$ & $4-\mathrm{OCH}_{3}(\mathbf{4 a})$ & $5 f$ & 53 \\
\hline 7 & $4-\mathrm{Cl}(\mathbf{3 g})$ & $4-\mathrm{OCH}_{3}(\mathbf{4 a})$ & $5 g$ & 38 \\
\hline 8 & 4-H (3a) & $4-\mathrm{NO}_{2}(\mathbf{4 b})$ & $5 \mathrm{~h}$ & 54 \\
\hline 9 & $4-\mathrm{OCH}_{3}(\mathbf{3 c})$ & $4-\mathrm{NO}_{2}(\mathbf{4 b})$ & $5 \mathbf{i}$ & 51 \\
\hline 10 & $4-\mathrm{F}(\mathbf{3 f})$ & $4-\mathrm{NO}_{2}(\mathbf{4 b})$ & $5 \mathbf{j}$ & 51 \\
\hline 11 & $4-\mathrm{OCH}_{3}(\mathbf{3 c})$ & $4-\mathrm{N}\left(\mathrm{CH}_{2} \mathrm{CH}_{2}\right)_{2} \mathrm{O}(4 \mathrm{c})$ & $5 \mathbf{k}$ & 52 \\
\hline 12 & $4-\mathrm{OCH}_{3}(\mathbf{3 c})$ & $3-\mathrm{OCH}_{3}(\mathbf{4 d})$ & 51 & 52 \\
\hline
\end{tabular}

${ }^{a}$ Reaction conditions: $\mathbf{3 a} \sim \mathbf{3 g}(1 \mathrm{mmol})$, iodobenzene derivatives $(3 \mathrm{mmol})$, KOAc $(3 \mathrm{mmol})$, DPPENT $(0.01 \mathrm{mmol}), \mathrm{Pd}(\mathrm{OAc})_{2}(0.05 \mathrm{mmol})$ in DMF $(4 \mathrm{~mL})$ at $120{ }^{\circ} \mathrm{C}, 24 \mathrm{~h} ;{ }^{b}$ Isolated yield.

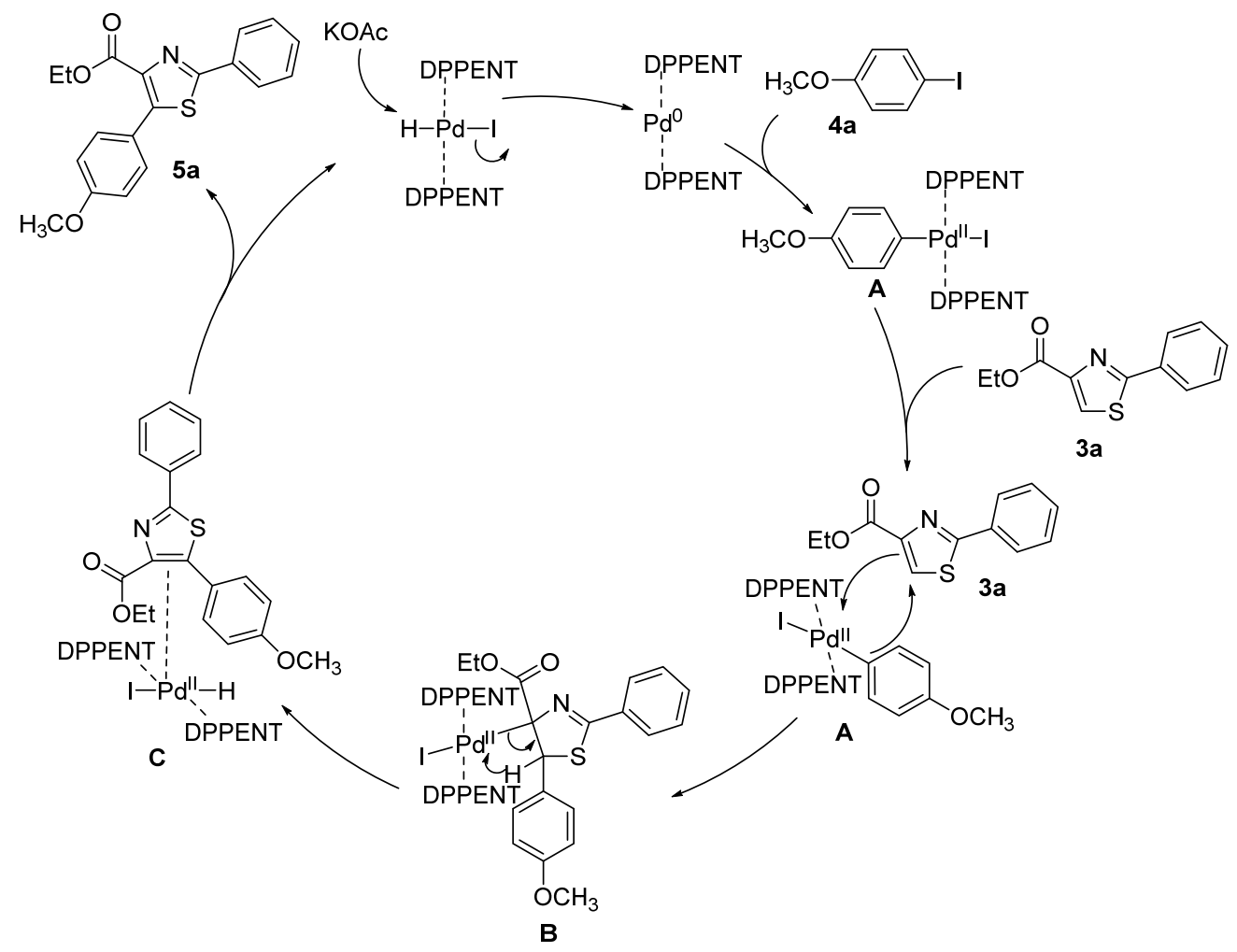

图式 2 反应机理推测

Scheme 2 Proposed reaction mechanism

\section{3 实验部分}

\section{1 仪器与试剂}

Bruker Avance III 400 型核磁共振仪(瑞士 Bruker 公
司), 以 $\mathrm{CDCl}_{3}$ 或二甲基亚砜(DMSO)为溶剂, $\mathrm{TMS}$ 为内 标; Waters Delta 600 型高效液相质谱仪(爱尔兰 Waters 公司); Agilent 6545 Q-TOF 高分辨质谱仪(美国 Agilent 公司); SGWX-4 显微熔点仪(上海精密科学仪器有限公 
司); 薄层色谱分析(TLC)在硅胶板上进行(硅胶板购于 德国 MACHEREY-NAGEL 公司).

硫代苯甲酰胺(2a) 商业购买, 含量为 $98 \%$ (质量分 数); DMF 含量为 $99.9 \%$ (质量分数), 四氢呋喃、无水乙 醇、乙酸乙酯和石油醚均为市售分析纯, 不做其他处理, 直接使用.

\section{2 实验方法}

\subsection{1 化合物 $\mathbf{2 b} \sim \mathbf{2 g}$ 的合成通法}

向取代苯甲酸 $6.57 \mathrm{mmol}, 1.0$ equiv.)的 DMF (5 mL) 溶液中分别加入 $N, N$-二异丙基乙胺(DIPEA) (26.28 mmol, 4.0 equiv.)、1-羟基苯并三唑(HOBT) $(9.86 \mathrm{mmol}$, 1.5 equiv.)和 1-乙基-(3-二甲基氨基丙基)碳酰二亚胺盐 酸盐(EDCI) (9.86 mmol, 1.5 equiv.), 室温摚拌反应 10 $\min$ 后, 再加入碳酸铵 (39.42 mmol, 6.0 equiv.), 继续反 应 $24 \mathrm{~h}$. 反应完毕, 将反应液倾倒至 $50 \mathrm{~mL}$ 蒸馏水中, 搅拌, 冷却析出晶体, 抽滤, 滤饼自然干燥. 滤液用乙 酸乙酯萃取 $(30 \mathrm{~mL} \times 3)$, 合并有机相并用饱和食盐水洗 涤(50 mL $\times 3)$, 无水硫酸钠干燥, 减压浓缩, 合并滤饼 与浓缩物得到取代苯甲酰胺, 不需纯化直接进入下一步 反应.

向取代苯甲酰胺 (1 mmol, 1 equiv.) 的四氢呋喃 (THF) (15 mL)溶液中加入 Lawesson 试剂(1.2 mmol, 1.2 equiv.), 于室温反应 $4 \mathrm{~h}$. 反应完毕, 将反应液冷却至 $0{ }^{\circ} \mathrm{C}$, 加入 $20 \mathrm{~mL}$ 饱和碳酸氢钠水溶液淬灭, 乙酸乙酯 萃取 $(25 \mathrm{~mL} \times 3)$, 合并有机相, 无水硫酸钠干燥, 过滤, 减压浓缩滤液, 浓缩物经柱层析(乙酸乙酯/石油醚)得到 产物取代硫代苯甲酰胺 $\mathbf{2 b} \sim 2 \mathrm{~g}$.

4-甲基硫代苯甲酰胺(2b): 黄色固体, 产率 $89 \%$. m.p. $114 \sim 116{ }^{\circ} \mathrm{C}$ (lit. ${ }^{[39]}$ m.p. $113 \sim 115{ }^{\circ} \mathrm{C}$ ); ${ }^{1} \mathrm{H}$ NMR $\left(400 \mathrm{MHz}, \mathrm{DMSO}-d_{6}\right) \delta: 9.79$ (s, $\left.1 \mathrm{H}, \mathrm{NH}\right), 9.43(\mathrm{~s}, 1 \mathrm{H}$, $\mathrm{NH}), 7.83(\mathrm{~d}, J=8.2 \mathrm{~Hz}, 2 \mathrm{H}, \operatorname{ArH}), 7.22(\mathrm{~d}, J=8.0 \mathrm{~Hz}$, 2H, $\mathrm{ArH}$ ), 2.34 (s, 3H, $\mathrm{CH}_{3}$ ); LC-MS m/z: $152.1[\mathrm{M}+\mathrm{H}]^{+}$.

4-甲氧基硫代苯甲酰胺(2c): 黄色固体, 产率 $86 \%$. m.p. $144 \sim 146{ }^{\circ} \mathrm{C}$ (lit. ${ }^{[39]}$ m.p. $145 \sim 147{ }^{\circ} \mathrm{C}$ ); ${ }^{1} \mathrm{H}$ NMR $\left(400 \mathrm{MHz}, \mathrm{DMSO}-d_{6}\right) \delta: 9.64(\mathrm{~s}, 1 \mathrm{H}, \mathrm{NH}), 9.32(\mathrm{~s}, 1 \mathrm{H}$, $\mathrm{NH}), 7.96(\mathrm{~d}, J=8.9 \mathrm{~Hz}, 2 \mathrm{H}, \mathrm{ArH}), 6.96(\mathrm{~d}, J=8.9 \mathrm{~Hz}$, $2 \mathrm{H}, \mathrm{ArH}), 3.82\left(\mathrm{~s}, 3 \mathrm{H}, \mathrm{OCH}_{3}\right.$ ); LC-MS $m / z: 168.0[\mathrm{M}+$ $\mathrm{H}]^{+}$.

3-甲氧基硫代苯甲酰胺(2d): 黄色固体, 产率 77\%. m.p. $96 \sim 98{ }^{\circ} \mathrm{C}$ (lit. ${ }^{[40]}$ m.p. $98 \sim 99.5{ }^{\circ} \mathrm{C}$ ); ${ }^{1} \mathrm{H}$ NMR $(400$ MHz, DMSO- $\left.d_{6}\right) \delta: 9.91(\mathrm{~s}, 1 \mathrm{H}, \mathrm{NH}), 9.50(\mathrm{~s}, 1 \mathrm{H}, \mathrm{NH})$, 7.47 (dd, $J=7.9,1.6 \mathrm{~Hz}, 1 \mathrm{H}, \mathrm{ArH}), 7.43$ (s, 1H, ArH), 7.33 (t, $J=8.0 \mathrm{~Hz}, 1 \mathrm{H}, A r H), 7.08(\mathrm{dd}, J=8.1,2.6 \mathrm{~Hz}, 1 \mathrm{H}$, $\mathrm{ArH}$ ), 3.79 (s, 3H, $\mathrm{OCH}_{3}$ ); LC-MS m/z: $168.0[\mathrm{M}+\mathrm{H}]^{+}$.

4-三氟甲氧基硫代苯甲酰胺 $(2 \mathbf{e})^{[41]}$ : 黄色固体, 产
率 98\%. m.p. $124 \sim 126{ }^{\circ} \mathrm{C} ;{ }^{1} \mathrm{H}$ NMR $(400 \mathrm{MHz}$, DMSO- $\left.d_{6}\right) \delta: 10.03(\mathrm{~s}, 1 \mathrm{H}, \mathrm{NH}), 9.62(\mathrm{~s}, 1 \mathrm{H}, \mathrm{NH}), 7.98(\mathrm{~d}$, $J=8.8 \mathrm{~Hz}, 2 \mathrm{H}, \mathrm{ArH}), 7.42$ (d, $J=7.8 \mathrm{~Hz}, 2 \mathrm{H}, \operatorname{ArH})$; LC-MS $m / z: 222.0[\mathrm{M}+\mathrm{H}]^{+}$.

4-氟硫代苯甲酰胺 $(\mathbf{2 f})$ : 黄色固体, 产率 $82 \%$. m.p. 146 $148{ }^{\circ} \mathrm{C}$ (lit. ${ }^{[39]}$ m.p. $143 \sim 145{ }^{\circ} \mathrm{C}$ ); ${ }^{1} \mathrm{H}$ NMR (400 MHz, DMSO- $\left.d_{6}\right) \delta: 9.92(\mathrm{~s}, 1 \mathrm{H}, \mathrm{NH}), 9.53(\mathrm{~s}, 1 \mathrm{H}, \mathrm{NH})$, $8.01 \sim 7.95(\mathrm{~m}, 2 \mathrm{H}, \operatorname{ArH}), 7.30 \sim 7.23(\mathrm{~m}, 2 \mathrm{H}, \operatorname{ArH})$; LC-MS $m / z: 156.0[\mathrm{M}+\mathrm{H}]^{+}$.

4-氯硫代苯甲酰胺 $(2 \mathrm{~g})$ : 黄色固体, 产率 79\%. m.p. $124 \sim 126{ }^{\circ} \mathrm{C}$ (lit. ${ }^{[39]}$ m.p. $\left.127 \sim 129{ }^{\circ} \mathrm{C}\right) ;{ }^{1} \mathrm{H}$ NMR $(400$ MHz, DMSO- $\left.d_{6}\right) \delta: 9.97(\mathrm{~s}, 1 \mathrm{H}, \mathrm{NH}), 9.57(\mathrm{~s}, 1 \mathrm{H}, \mathrm{NH})$, 7.90 (d, $J=8.6 \mathrm{~Hz}, 2 \mathrm{H}, \mathrm{ArH}), 7.49$ (d, $J=8.6 \mathrm{~Hz}, 2 \mathrm{H}$, $\mathrm{ArH})$; LC-MS $m / z$ : $172.0[\mathrm{M}+\mathrm{H}]^{+}$.

3.2.2 化合物 $\mathbf{3 a} \sim \mathbf{3 g}$ 的合成通法

将取代硫代苯甲酰胺 $\mathbf{2 a} \sim 2 \mathbf{g}$ (1 mmol, 1 equiv.)溶解 于无水乙醇 $(3 \mathrm{~mL})$ 中, 再加入澳代丙酮酸乙酯 $(1.2$ $\mathrm{mmol}, 1.2$ equiv.), 于室温反应 $0.5 \mathrm{~h}$ 后, 升温至 $78{ }^{\circ} \mathrm{C}$ 反 应 $2 \mathrm{~h}$. 反应完毕, 待反应液冷却至室温后, 加入乙酸乙 酯(15 mL) 稀释, 分别用饱和碳酸氢钠水溶液 $(30 \mathrm{~mL} \times$ $3)$ 和饱和氯化钠水溶液 $(30 \mathrm{~mL} \times 3)$ 洗涤, 有机相经无水 硫酸钠干燥, 过滤, 减压浓缩滤液, 浓缩物经柱层析(乙 酸乙酯/石油醚)得到产物 $3 \mathrm{a} \sim 3 \mathrm{~g}$.

2-苯基噻唑-4-甲酸乙酯 $(3 \mathbf{a})^{[42]}$ : 黄色油状物, 收率 $78 \%$. ${ }^{1} \mathrm{H}$ NMR (400 MHz, DMSO- $\left.d_{6}\right) \delta: 8.59$ (s, $\left.1 \mathrm{H}, \mathrm{ArH}\right)$, $8.00(\mathrm{dd}, J=6.7,3.0 \mathrm{~Hz}, 2 \mathrm{H}, \operatorname{ArH}), 7.57 \sim 7.53(\mathrm{~m}, 3 \mathrm{H}$, $\operatorname{ArH}), 4.35$ (q, $\left.J=7.1 \mathrm{~Hz}, 2 \mathrm{H}, \mathrm{OCH}_{2}\right), 1.34$ (t, $J=7.1 \mathrm{~Hz}$, $\left.3 \mathrm{H}, \mathrm{CH}_{2} \mathrm{CH}_{3}\right)$; LC-MS $m / z: 234.1[\mathrm{M}+\mathrm{H}]^{+}$.

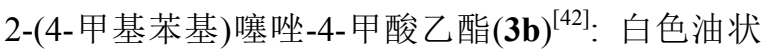
物, 收率 $67 \% .{ }^{1} \mathrm{H}$ NMR (400 MHz, DMSO- $\left.d_{6}\right) \delta: 8.55$ (s, $1 \mathrm{H}, \operatorname{ArH}), 7.88(\mathrm{~d}, J=8.2 \mathrm{~Hz}, 2 \mathrm{H}, \operatorname{ArH}), 7.35$ (d, $J=7.7$ $\mathrm{Hz}, 2 \mathrm{H}, \mathrm{ArH}), 4.34$ (q, $\left.J=7.1 \mathrm{~Hz}, 2 \mathrm{H}, \mathrm{OCH}_{2}\right), 2.38(\mathrm{~s}, 3 \mathrm{H}$, $\mathrm{CH}_{3}$ ), 1.33 (t, $J=7.1 \mathrm{~Hz}, 3 \mathrm{H}, \mathrm{CH}_{2} \mathrm{CH}_{3}$ ); LC-MS $m / z: 248.1$ $[\mathrm{M}+\mathrm{H}]^{+}$.

2-(4-甲氧基苯基)噻唑-4-甲酸乙酯(3c)：白色固体， 产率 65\%. m.p. 92 94 ${ }^{\circ} \mathrm{C}$ (lit. ${ }^{[42]}$ m.p. 91 93 ${ }^{\circ} \mathrm{C}$ ); ${ }^{1} \mathrm{H}$ NMR (400 MHz, DMSO- $\left.d_{6}\right) \delta: 8.49$ (s, $\left.1 \mathrm{H}, \mathrm{ArH}\right), 7.92(\mathrm{~d}$, $J=8.9 \mathrm{~Hz}, 2 \mathrm{H}, \mathrm{ArH}), 7.08$ (d, $J=8.8 \mathrm{~Hz}, 2 \mathrm{H}, \mathrm{ArH}), 4.33$ (q, $\left.J=7.1 \mathrm{~Hz}, 2 \mathrm{H}, \mathrm{OCH}_{2}\right), 3.84\left(\mathrm{~s}, 3 \mathrm{H}, \mathrm{OCH}_{3}\right), 1.33(\mathrm{t}, J=$ $\left.7.1 \mathrm{~Hz}, 3 \mathrm{H}, \mathrm{CH}_{2} \mathrm{CH}_{3}\right)$; LC-MS m/z: $264.1[\mathrm{M}+\mathrm{H}]^{+}$.

2-(3-甲氧基苯基)噻唑-4-甲酸乙酯(3d): 白色固体, 产率 74\%. m.p. 58 60 ${ }^{\circ} \mathrm{C}$ (lit. ${ }^{[43]}$ m.p. $58{ }^{\circ} \mathrm{C}$ ); ${ }^{1} \mathrm{H}$ NMR $\left(400 \mathrm{MHz}, \mathrm{CDCl}_{3}\right) \delta: 8.18$ (s, $\left.1 \mathrm{H}, \mathrm{ArH}\right), 7.61$ (dd, $J=2.6$, $1.6 \mathrm{~Hz}, 1 \mathrm{H}, \mathrm{ArH}$ ), 7.57 (ddd, $J=7.7,1.7,1.0 \mathrm{~Hz}, 1 \mathrm{H}$, ArH), 7.38 (t, $J=7.9 \mathrm{~Hz}, 1 \mathrm{H}, \mathrm{ArH}), 7.03$ (ddd, $J=8.3,2.6$, 
$1.0 \mathrm{~Hz}, 1 \mathrm{H}, \mathrm{ArH}$ ), 4.47 (q, J=7.1 Hz, 2H, $\mathrm{OCH}_{2}$ ), 3.92 (s, $\left.3 \mathrm{H}, \mathrm{OCH}_{3}\right), 1.46$ (t, $J=7.1 \mathrm{~Hz}, 3 \mathrm{H}, \mathrm{CH}_{2} \mathrm{CH}_{3}$ ); LC-MS $m / z$ : $264.1[\mathrm{M}+\mathrm{H}]^{+}$.

2-(4-三氟甲氧基苯基)噻唑-5-甲酸乙酯 (3e) ${ }^{[43]}$ : 白 色固体, 收率 92\%. m.p. 88 90 ${ }^{\circ} \mathrm{C} ;{ }^{1} \mathrm{H}$ NMR $(400 \mathrm{MHz}$, DMSO- $\left.d_{6}\right) \delta: 8.62(\mathrm{~s}, 1 \mathrm{H}, \mathrm{ArH}), 8.13(\mathrm{~d}, J=8.8 \mathrm{~Hz}, 2 \mathrm{H}$, ArH), 7.54 (dd, $J=8.9,1.1 \mathrm{~Hz}, 2 \mathrm{H}, \mathrm{ArH}), 4.35$ (q, $J=7.1$ $\mathrm{Hz}, 2 \mathrm{H}, \mathrm{OCH}_{2}$ ), 1.34 (t, $J=7.1 \mathrm{~Hz}, 3 \mathrm{H}, \mathrm{CH}_{2} \mathrm{CH}_{3}$ ); LC-MS $m / z: 318.0[\mathrm{M}+\mathrm{H}]^{+}$.

2-(4-氟苯基)噻唑-4-甲酸乙酯(3f): 白色固体, 收率 75\%. m.p. 69 71 ${ }^{\circ} \mathrm{C}$ (lit. ${ }^{[44]}$ m.p. $70 \sim 72{ }^{\circ} \mathrm{C}$ ); ${ }^{1} \mathrm{H}$ NMR $\left(400 \mathrm{MHz}\right.$, DMSO- $\left.d_{6}\right) \delta: 8.59$ (s, $\left.1 \mathrm{H}, \mathrm{ArH}\right), 8.05$ (dd, $J=$ 8.9, $5.3 \mathrm{~Hz}, 2 \mathrm{H}, \mathrm{ArH}), 7.39$ (t, $J=8.8 \mathrm{~Hz}, 2 \mathrm{H}, \mathrm{ArH}), 4.34$ (q, $\left.J=7.1 \mathrm{~Hz}, 2 \mathrm{H}, \mathrm{OCH}_{2}\right), 1.34(\mathrm{t}, J=7.1 \mathrm{~Hz}, 3 \mathrm{H}$, $\mathrm{CH}_{2} \mathrm{CH}_{3}$ ); LC-MS $m / z: 252.0[\mathrm{M}+\mathrm{H}]^{+}$.

2-(4-氯苯基)噻唑-4-甲酸乙酯 (3g): 白色固体, 产率 73\%. m.p. 88 90 ${ }^{\circ} \mathrm{C}$ (lit. ${ }^{[44]}$ m.p. $88 \sim 89{ }^{\circ} \mathrm{C}$ ); ${ }^{1} \mathrm{H}$ NMR $\left(400 \mathrm{MHz}, \mathrm{DMSO}-d_{6}\right) \delta: 8.61(\mathrm{~s}, 1 \mathrm{H}, \mathrm{ArH}), 8.01$ (d, $J=8.6$ $\mathrm{Hz}, 2 \mathrm{H}, \mathrm{ArH}), 7.61$ (d, $J=8.6 \mathrm{~Hz}, 2 \mathrm{H}, \mathrm{ArH}), 4.34$ (q, $J=$ $\left.7.1 \mathrm{~Hz}, 2 \mathrm{H}, \mathrm{OCH}_{2}\right), 1.33\left(\mathrm{t}, J=7.1 \mathrm{~Hz}, 3 \mathrm{H}, \mathrm{CH}_{2} \mathrm{CH}_{3}\right)$; LC-MS $m / z: 268.0[\mathrm{M}+\mathrm{H}]^{+}$.

\section{2 .3 化合物 $\mathbf{5 a} \sim \mathbf{5}$ 的合成通法}

氩气保护下，向化合物 $\mathbf{3 a} \sim \mathbf{3 g}(1 \mathrm{mmol}, 1$ equiv.) 的 DMF (4 mL)溶液中依次加入取代碘苯 $4 \mathbf{a} \sim 4 \mathbf{d}(3 \mathrm{mmol}$, 3 equiv.)、DPPENT (0.01 mmol, 0.01 equiv.)、醋酸钯 ( $0.05 \mathrm{mmol}, 0.05$ equiv.) 和醋酸钾 ( $3 \mathrm{mmol}, 3$ equiv.), 于 $120{ }^{\circ} \mathrm{C}$ 下反应 $24 \mathrm{~h}$. 反应完毕, 待反应液冷却至室温, 加入乙酸乙酯 $(25 \mathrm{~mL})$ 稀释, 用饱和食盐水洗涤 $(50 \mathrm{~mL}$ $\times 3)$, 有机相经无水硫酸钠干燥、过滤、减压浓缩滤液, 浓缩物经柱层析( 乙酸乙酯/石油醚)得到产物 $5 \mathbf{a} \sim 51$.

2-苯基-5-(4-甲氧基苯基)噻唑-4-甲酸乙酯 (5a)：白 色固体, 收率 48\%. m.p. $125 \sim 127{ }^{\circ} \mathrm{C}$ (lit. ${ }^{[45]}$ m.p. 126 $\left.127{ }^{\circ} \mathrm{C}\right) ;{ }^{1} \mathrm{H}$ NMR $\left(400 \mathrm{MHz}, \mathrm{CDCl}_{3}\right) \delta: 8.01$ (dd, $J=6.6$, $2.9 \mathrm{~Hz}, 2 \mathrm{H}, \mathrm{ArH}), 7.51$ (d, $J=8.6 \mathrm{~Hz}, 2 \mathrm{H}, \mathrm{ArH}), 7.49 \sim$ $7.46(\mathrm{~m}, 3 \mathrm{H}, \mathrm{ArH}), 6.98(\mathrm{~d}, J=8.6 \mathrm{~Hz}, 2 \mathrm{H}, \operatorname{ArH}), 4.36(\mathrm{q}$, $\left.J=7.1 \mathrm{~Hz}, 2 \mathrm{H}, \mathrm{OCH}_{2}\right), 3.89\left(\mathrm{~s}, 3 \mathrm{H}, \mathrm{OCH}_{3}\right), 1.31(\mathrm{t}, J=7.2$ $\left.\mathrm{Hz}, 3 \mathrm{H}, \mathrm{CH}_{2} \mathrm{CH}_{3}\right) ;{ }^{13} \mathrm{C} \mathrm{NMR}\left(101 \mathrm{MHz}, \mathrm{CDCl}_{3}\right) \delta: 164.47$, $161.36,159.35,145.16,139.65,131.71,130.28,129.50$, $127.91,125.73,121.47,112.60,60.30,54.34,13.11$; HRMS (ESI) calcd for $\mathrm{C}_{19} \mathrm{H}_{18} \mathrm{NO}_{3} \mathrm{~S}[\mathrm{M}+\mathrm{H}]^{+} 340.1007$, found 340.0995 .

2-(4-甲基苯基)-5-(4-甲氧基苯基)噻唑-4-甲酸乙酯 (5b): 白色固体, 收率 54\%. m.p. 106 108 ${ }^{\circ} \mathrm{C} ;{ }^{1} \mathrm{H}$ NMR $\left(400 \mathrm{MHz}, \mathrm{DMSO}-d_{6}\right) \delta: 7.85(\mathrm{~d}, J=8.1 \mathrm{~Hz}, 2 \mathrm{H}, \mathrm{ArH})$, $7.50(\mathrm{~d}, J=8.7 \mathrm{~Hz}, 2 \mathrm{H}, \mathrm{ArH}), 7.35$ (d, $J=8.1 \mathrm{~Hz}, 2 \mathrm{H}$,
ArH), 7.03 (d, $J=8.8 \mathrm{~Hz}, 2 \mathrm{H}, \operatorname{ArH}), 4.22(\mathrm{q}, J=7.1 \mathrm{~Hz}$, $\left.2 \mathrm{H}, \mathrm{OCH}_{2}\right), 3.82\left(\mathrm{~s}, 3 \mathrm{H}, \mathrm{OCH}_{3}\right), 2.38\left(\mathrm{~s}, 3 \mathrm{H}, \mathrm{CH}_{3}\right), 1.18$ (t, $\left.J=7.1 \mathrm{~Hz}, 3 \mathrm{H}, \mathrm{CH}_{2} \mathrm{CH}_{3}\right) ;{ }^{13} \mathrm{C}$ NMR $\left(101 \mathrm{MHz}, \mathrm{CDCl}_{3}\right) \delta$ : $165.74,162.46,160.35,145.75,140.92,140.55,131.32$, $130.13,129.64,126.70,122.64,113.63,61.32,55.39$, 21.52, 14.17; HRMS (ESI) calcd for $\mathrm{C}_{20} \mathrm{H}_{20} \mathrm{NO}_{3} \mathrm{~S}[\mathrm{M}+$ $\mathrm{H}]^{+} 354.1164$, found 354.1149 .

2-(4-甲氧基苯基)-5-(4-甲氧基苯基)噻唑-4-甲酸乙 酯(5c): 白色固体, 收率 51\%. m.p. 94 96 ${ }^{\circ} \mathrm{C} ;{ }^{1} \mathrm{H}$ NMR $\left(400 \mathrm{MHz}, \mathrm{DMSO}-d_{6}\right) \delta: 7.90(\mathrm{~d}, J=8.8 \mathrm{~Hz}, 2 \mathrm{H}, \mathrm{ArH})$, 7.49 (d, $J=8.8 \mathrm{~Hz}, 2 \mathrm{H}, \operatorname{ArH}), 7.09$ (d, $J=8.9 \mathrm{~Hz}, 2 \mathrm{H}$, $\operatorname{Ar} H), 7.03$ (d, $J=8.8 \mathrm{~Hz}, 2 \mathrm{H}, \operatorname{ArH}), 4.21(\mathrm{q}, J=7.1 \mathrm{~Hz}$, $\left.2 \mathrm{H}, \mathrm{OCH}_{2}\right), 3.84\left(\mathrm{~s}, 3 \mathrm{H}, \mathrm{OCH}_{3}\right), 3.82\left(\mathrm{~s}, 3 \mathrm{H}, \mathrm{OCH}_{3}\right), 1.17$ (t, $\left.J=7.1 \mathrm{~Hz}, 3 \mathrm{H}, \mathrm{CH}_{2} \mathrm{CH}_{3}\right) ;{ }^{13} \mathrm{C}$ NMR $\left(101 \mathrm{MHz}, \mathrm{CDCl}_{3}\right)$ $\delta: 165.60,162.33,161.64,160.36,145.29,140.19,131.32$, $128.43,125.44,122.56,114.30,113.64,61.37,55.47$, 55.40, 14.16; HRMS (ESI) calcd for $\mathrm{C}_{20} \mathrm{H}_{20} \mathrm{NO}_{4} \mathrm{~S}[\mathrm{M}+$ $\mathrm{H}]^{+}$370.1113, found 370.1099 .

2-(3-甲氧基基苯基)-5-(4-甲氧基苯基)噻唑-4-甲酸 乙酯(5d): 淡黄色固体，收率 50\%. m.p. $105 \sim 107{ }^{\circ} \mathrm{C}$; ${ }^{1} \mathrm{H}$ NMR (400 MHz, $\left.\mathrm{CDCl}_{3}\right) \delta: 7.58(\mathrm{dd}, J=2.6,1.6 \mathrm{~Hz}$, $1 \mathrm{H}, \mathrm{ArH}$ ), 7.55 (ddd, $J=7.7,1.7,1.0 \mathrm{~Hz}, 1 \mathrm{H}, \mathrm{ArH}), 7.51$ (d, $J=8.8 \mathrm{~Hz}, 2 \mathrm{H}, \mathrm{ArH}), 7.38$ (t, $J=7.9 \mathrm{~Hz}, 1 \mathrm{H}, \mathrm{ArH})$, 7.02 (ddd, $J=8.3,2.6,0.9 \mathrm{~Hz}, 1 \mathrm{H}, \mathrm{ArH}$ ), 6.98 (d, $J=8.9$ $\mathrm{Hz}, 2 \mathrm{H}, \mathrm{ArH}), 4.35$ (q, $\left.J=7.1 \mathrm{~Hz}, 2 \mathrm{H}, \mathrm{OCH}_{2}\right), 3.92(\mathrm{~s}, 3 \mathrm{H}$, $\left.\mathrm{OCH}_{3}\right), 3.89\left(\mathrm{~s}, 3 \mathrm{H}, \mathrm{OCH}_{3}\right), 1.31(\mathrm{t}, J=7.1 \mathrm{~Hz}, 3 \mathrm{H}$, $\left.\mathrm{CH}_{2} \mathrm{CH}_{3}\right) ;{ }^{13} \mathrm{C}$ NMR $\left(101 \mathrm{MHz}, \mathrm{CDCl}_{3}\right) \delta: 165.40,162.43$, $160.42,160.00,146.28,140.67,134.04,131.34,130.00$, 122.51, 119.42, 116.87, 113.66, 111.36, 61.37, 55.55, 55.40, 14.16; HRMS (ESI) calcd for $\mathrm{C}_{20} \mathrm{H}_{20} \mathrm{NO}_{4} \mathrm{~S}[\mathrm{M}+$ $\mathrm{H}]^{+} 370.1113$, found 370.1100 .

2-(4-(三氟甲氧基)苯基)-5-(4-甲氧基苯基)噻唑-4甲酸乙酯(5e): 白色固体, 产率 55\%. m.p. 97 99 ${ }^{\circ} \mathrm{C} ;{ }^{1} \mathrm{H}$ NMR $\left(400 \mathrm{MHz}\right.$, DMSO- $\left.d_{6}\right) \delta: 8.09(\mathrm{~d}, J=8.8 \mathrm{~Hz}, 2 \mathrm{H}$, $\operatorname{ArH}), 7.57 \sim 7.49$ (m, 4H, ArH), 7.04 (d, $J=8.8 \mathrm{~Hz}, 2 \mathrm{H}$, $\operatorname{ArH}), 4.23$ (q, $\left.J=7.1 \mathrm{~Hz}, 2 \mathrm{H}, \mathrm{OCH}_{2}\right), 3.82\left(\mathrm{~s}, 3 \mathrm{H}, \mathrm{OCH}_{3}\right.$ ), $1.18\left(\mathrm{t}, J=7.1 \mathrm{~Hz}, 3 \mathrm{H}, \mathrm{CH}_{2} \mathrm{CH}_{3}\right) ;{ }^{13} \mathrm{C}$ NMR $(101 \mathrm{MHz}$, $\left.\mathrm{CDCl}_{3}\right) \delta: 162.63,161.28,159.46,149.62,145.60,139.94$, $130.38,130.29,127.25,121.23,120.61,120.17,112.65$, 60.36, 54.35, 13.09; HRMS (ESI) calcd for $\mathrm{C}_{20} \mathrm{H}_{17} \mathrm{NO}_{4} \mathrm{~S}$ $[\mathrm{M}+\mathrm{H}]^{+}$424.0830, found 424.0814.

2-(4-氟苯基)-5-(4-甲氧基苯基)噻唑-4-甲酸乙酯 (5f): 白色固体, 收率 54\%. m.p. 162 164 ${ }^{\circ} \mathrm{C} ;{ }^{1} \mathrm{H}$ NMR $\left(400 \mathrm{MHz}, \mathrm{DMSO}-d_{6}\right) \delta: 8.03(\mathrm{dd}, J=8.8,5.3 \mathrm{~Hz}, 2 \mathrm{H}$, $\operatorname{ArH}), 7.52(\mathrm{~d}, J=8.8 \mathrm{~Hz}, 2 \mathrm{H}, \operatorname{ArH}), 7.40(\mathrm{t}, J=8.8 \mathrm{~Hz}$, 
2H, ArH), 7.04 (d, $J=8.8 \mathrm{~Hz}, 2 \mathrm{H}, \operatorname{ArH}), 4.23$ (q, $J=7.1$ $\mathrm{Hz}, 2 \mathrm{H}, \mathrm{OCH}_{2}$ ), 3.83 (s, $\left.3 \mathrm{H}, \mathrm{OCH}_{3}\right), 1.18$ (t, $J=7.1 \mathrm{~Hz}$, $\left.3 \mathrm{H}, \mathrm{CH}_{2} \mathrm{CH}_{3}\right) ;{ }^{13} \mathrm{C}$ NMR $\left(101 \mathrm{MHz}, \mathrm{CDCl}_{3}\right) \delta: 164.24$, 164.14 (d, $J=252.5 \mathrm{~Hz}$ ), 162.38, 160.44, 146.24, 140.78, 131.33, $129.20(\mathrm{~d}, J=3.0 \mathrm{~Hz}), 128.74(\mathrm{~d}, J=9.1 \mathrm{~Hz})$, 122.40, 116.10 (d, $J=22.2 \mathrm{~Hz}), 113.67,61.37,55.40$, 14.16; HRMS (ESI) calcd for $\mathrm{C}_{19} \mathrm{H}_{16} \mathrm{FNNaO}_{3} \mathrm{~S}[\mathrm{M}+\mathrm{Na}]^{+}$ 380.0733 , found 380.0718 .

2-(4-氯苯基)-5-(4-甲氧基苯基)噻唑-4-甲酸乙酯 (5g): 白色固体, 收率 39\%. m.p. $134 \sim 136{ }^{\circ} \mathrm{C} ;{ }^{1} \mathrm{H}$ NMR (400 MHz, DMSO- $\left.d_{6}\right) \delta: 7.99$ (d, $\left.J=8.6 \mathrm{~Hz}, 2 \mathrm{H}, \mathrm{ArH}\right)$, $7.62(\mathrm{~d}, J=8.5 \mathrm{~Hz}, 2 \mathrm{H}, \mathrm{ArH}), 7.52(\mathrm{~d}, J=8.8 \mathrm{~Hz}, 2 \mathrm{H}$, ArH), 7.05 (d, $J=8.8 \mathrm{~Hz}, 2 \mathrm{H}, \operatorname{ArH}), 4.23$ (q, $J=7.1 \mathrm{~Hz}$, $\left.2 \mathrm{H}, \mathrm{OCH}_{2}\right), 3.83\left(\mathrm{~s}, 3 \mathrm{H}, \mathrm{OCH}_{3}\right), 1.18(\mathrm{t}, J=7.1 \mathrm{~Hz}, 3 \mathrm{H}$, $\left.\mathrm{CH}_{2} \mathrm{CH}_{3}\right) ;{ }^{13} \mathrm{C} \mathrm{NMR}\left(101 \mathrm{MHz}, \mathrm{CDCl}_{3}\right) \delta: 163.00,161.28$, $159.43,145.42,139.84,135.47,130.27,130.26,128.16$, 126.88, 121.27, 112.63, 60.35, 54.35, 13.10; HRMS (ESI) calcd for $\mathrm{C}_{19} \mathrm{H}_{17} \mathrm{ClNO}_{3} \mathrm{~S}[\mathrm{M}+\mathrm{H}]^{+} 374.0618$, found 374.0605 .

2-苯基-5-(4-硝基苯基)噻唑-4-甲酸乙酯 $(\mathbf{5 h}$ ): 淡黄 色固体, 收率 54\%. m.p. 145 147 ${ }^{\circ} \mathrm{C} ;{ }^{1} \mathrm{H}$ NMR (400 $\left.\mathrm{MHz}, \mathrm{CDCl}_{3}\right) \delta: 8.24(\mathrm{~d}, J=8.7 \mathrm{~Hz}, 2 \mathrm{H}, \mathrm{ArH}), 7.96 \sim 7.91$ (m, 2H, ArH), $7.66(\mathrm{~d}, J=8.7 \mathrm{~Hz}, 2 \mathrm{H}, \mathrm{ArH}), 7.43 \sim 7.40$ (m, 3H, ArH), 4.27 (q, $\left.J=7.1 \mathrm{~Hz}, 2 \mathrm{H}, \mathrm{OCH}_{2}\right), 1.22$ (t, $J=$ $\left.7.1 \mathrm{~Hz}, 3 \mathrm{H}, \mathrm{CH}_{2} \mathrm{CH}_{3}\right) ;{ }^{13} \mathrm{C}$ NMR (101 MHz, $\left.\mathrm{CDCl}_{3}\right) \delta$ : $166.44,160.79,146.95,141.56,141.42,136.24,131.24$, $130.11,130.00,128.09,127.31,125.86,123.36,122.35$, 60.73, 13.06; HRMS (ESI) calcd for $\mathrm{C}_{19} \mathrm{H}_{17} \mathrm{ClNO}_{3} \mathrm{~S}[\mathrm{M}+$ $\mathrm{H}]^{+}$355.0753, found 355.0738 .

2-(4-甲氧基苯基)-5-(4-硝基苯基)噻唑-4-甲酸乙酯 (5i): 黄色固体, 产率 52\%. m.p. $144 \sim 146{ }^{\circ} \mathrm{C} ;{ }^{1} \mathrm{H}$ NMR (400 MHz, DMSO- $\left.d_{6}\right) \delta: 8.32(\mathrm{~d}, J=8.7 \mathrm{~Hz}, 2 \mathrm{H}, \mathrm{ArH})$, $7.95(\mathrm{~d}, J=8.8 \mathrm{~Hz}, 2 \mathrm{H}, \operatorname{ArH}), 7.86(\mathrm{~d}, J=8.8 \mathrm{~Hz}, 2 \mathrm{H}$, $\operatorname{Ar} H), 7.12(\mathrm{~d}, J=8.8 \mathrm{~Hz}, 2 \mathrm{H}, \mathrm{ArH}), 4.22(\mathrm{q}, J=7.1 \mathrm{~Hz}$, $\left.2 \mathrm{H}, \mathrm{OCH}_{2}\right), 3.85\left(\mathrm{~s}, 3 \mathrm{H}, \mathrm{OCH}_{3}\right), 1.15(\mathrm{t}, J=7.1 \mathrm{~Hz}, 3 \mathrm{H}$, $\left.\mathrm{CH}_{2} \mathrm{CH}_{3}\right) ;{ }^{13} \mathrm{C}$ NMR (101 MHz, $\left.\mathrm{CDCl}_{3}\right) \delta: 166.38,160.95$, $160.87,146.85,141.15,140.61,136.42,129.96,127.47$, $124.08,122.32,113.38,60.69,54.46,13.05$; HRMS (ESI) calcd for $\mathrm{C}_{19} \mathrm{H}_{17} \mathrm{~N}_{2} \mathrm{O}_{5} \mathrm{~S} \quad[\mathrm{M}+\mathrm{H}]^{+} 385.0858$, found 385.0842 .

2-(4-氟苯基)-5-(4-硝基苯基)噻唑-4-甲酸乙酯(5j): 淡黄色固体, 产率 51\%. m.p. $162 \sim 164{ }^{\circ} \mathrm{C} ;{ }^{1} \mathrm{H}$ NMR $\left(400 \mathrm{MHz}, \mathrm{CDCl}_{3}\right) \delta: 8.24$ (d, J=8.8 Hz, 2H, ArH), 7.93 (dd, $J=8.8,5.2 \mathrm{~Hz}, 2 \mathrm{H}, \mathrm{ArH}), 7.65(\mathrm{~d}, J=8.8 \mathrm{~Hz}, 2 \mathrm{H}$, $\operatorname{ArH}), 7.11$ (t, $J=8.6 \mathrm{~Hz}, 2 \mathrm{H}, \operatorname{ArH}), 4.27$ (q, $J=7.1 \mathrm{~Hz}$, $\left.2 \mathrm{H}, \mathrm{OCH}_{2}\right), 1.21\left(\mathrm{t}, J=7.1 \mathrm{~Hz}, 3 \mathrm{H}, \mathrm{CH}_{2} \mathrm{CH}_{3}\right) ;{ }^{13} \mathrm{C} \mathrm{NMR}$ $\left(101 \mathrm{MHz}, \mathrm{CDCl}_{3}\right) \delta: 165.17,163.44(\mathrm{~d}, J=253.5 \mathrm{~Hz})$, 160.70, 147.00, 141.55, 141.44, 136.07, 130.00, $127.92(\mathrm{~d}$, $J=9.1 \mathrm{~Hz}), 127.61$ (d, $J=3.0 \mathrm{~Hz}), 122.37,115.29$ (d, $J=$ $22.2 \mathrm{~Hz}$ ), 60.77, 13.05; HRMS (ESI) calcd for $\mathrm{C}_{18} \mathrm{H}_{14} \mathrm{~F}-$ $\mathrm{NO}_{4} \mathrm{~S}[\mathrm{M}+\mathrm{H}]^{+} 373.0658$, found 373.0644 .

2-(4-甲氧基苯基)-5-(4-吗啉基苯基)噻唑-4-甲酸乙 酯(5k): 淡黄色固体, 产率 52\%. m.p. $96 \sim 98{ }^{\circ} \mathrm{C} ;{ }^{1} \mathrm{H}$ NMR (400 MHz, DMSO- $\left.d_{6}\right) \delta: 7.89(\mathrm{~d}, J=8.4 \mathrm{~Hz}, 2 \mathrm{H}$, ArH), $7.42(\mathrm{~d}, J=8.3 \mathrm{~Hz}, 2 \mathrm{H}, \mathrm{ArH}), 7.08(\mathrm{~d}, J=8.3 \mathrm{~Hz}$, $2 \mathrm{H}, \operatorname{ArH}), 7.01(\mathrm{~d}, J=8.4 \mathrm{~Hz}, 2 \mathrm{H}, \operatorname{ArH}), 4.23$ (q, $J=7.1$ $\left.\mathrm{Hz}, 2 \mathrm{H}, \mathrm{OCH}_{2}\right), 3.84\left(\mathrm{~s}, 3 \mathrm{H}, \mathrm{OCH}_{3}\right), 3.78 \sim 3.74[\mathrm{~m}, 4 \mathrm{H}$, $\left.\mathrm{N}\left(\mathrm{CH}_{2}\right)_{2}\right], 3.21 \sim 3.19\left[\mathrm{~m}, 4 \mathrm{H}, \mathrm{O}\left(\mathrm{CH}_{2}\right)_{2}\right], 1.20(\mathrm{t}, J=7.1$ $\left.\mathrm{Hz}, 3 \mathrm{H}, \mathrm{CH}_{2} \mathrm{CH}_{3}\right) ;{ }^{13} \mathrm{C} \mathrm{NMR}\left(101 \mathrm{MHz}, \mathrm{CDCl}_{3}\right) \delta: 164.16$, $161.55,160.87,160.41,139.17,130.05,127.52,127.21$, $124.71,124.16,113.31,113.18,65.43,60.23,54.40,48.03$, 13.17; HRMS (ESI) calcd for $\mathrm{C}_{23} \mathrm{H}_{24} \mathrm{~N}_{2} \mathrm{NaO}_{4} \mathrm{~S}[\mathrm{M}+\mathrm{Na}]^{+}$ 447.1354, found 447.1341.

2-(4-甲氧基苯基)-5-(3-甲氧基苯基)噻唑-4-甲酸乙 酯(5I): 白色固体，产率 51\%. m.p. $107 \sim 109{ }^{\circ} \mathrm{C} ;{ }^{1} \mathrm{H}$ NMR (400 MHz, $\left.\mathrm{CDCl}_{3}\right) \delta: 7.95(\mathrm{~d}, J=8.8 \mathrm{~Hz}, 2 \mathrm{H}, \mathrm{ArH})$, $7.35(\mathrm{t}, J=7.9 \mathrm{~Hz}, 1 \mathrm{H}, \mathrm{ArH}), 7.12(\mathrm{dt}, J=7.8,1.2 \mathrm{~Hz}, 1 \mathrm{H}$, ArH), 7.09 (dd, $J=2.5,1.6 \mathrm{~Hz}, 1 \mathrm{H}, \mathrm{ArH}), 7.01 \sim 6.96(\mathrm{~m}$, $3 \mathrm{H}, \mathrm{ArH}), 4.33$ (q, $\left.J=7.1 \mathrm{~Hz}, 2 \mathrm{H}, \mathrm{OCH}_{2}\right), 3.89(\mathrm{~s}, 3 \mathrm{H}$, $\left.\mathrm{OCH}_{3}\right), 3.87\left(\mathrm{~s}, 3 \mathrm{H}, \mathrm{OCH}_{3}\right), 1.28(\mathrm{~d}, J=7.1 \mathrm{~Hz}, 3 \mathrm{H}$, $\left.\mathrm{CH}_{2} \mathrm{CH}_{3}\right) ;{ }^{13} \mathrm{C} \mathrm{NMR}\left(101 \mathrm{MHz}, \mathrm{CDCl}_{3}\right) \delta: 166.15,162.31$, $161.67,159.21,144.47,141.12,131.73,129.24,128.44$, $125.47,122.35,115.53,114.67,114.30,61.38,55.47$, $55.37,14.05,1.06$; HRMS (ESI) calcd for $\mathrm{C}_{20} \mathrm{H}_{20} \mathrm{NO}_{4} \mathrm{~S}$ $[\mathrm{M}+\mathrm{H}]^{+}$370.1113, found 370.1099.)

辅助材料(Supporting Information) 目标化合物 5a $5 \mathbf{I}$ 的 ${ }^{1} \mathrm{H}$ NMR 和 ${ }^{13} \mathrm{C}$ NMR 谱图. 这些材料可以免费从本 刊网站(http://sioc-journal.cn/)上下载.

\section{References}

[1] Aggarwal, R.; Kumar, R.; Kumar, S.; Garg, G.; Mahajan, R.; Sharma, J. J. Fluorine Chem. 2011, 132, 965.

[2] Pawar, C. D.; Sarkate, A. P.; Karnik, K. S.; Bahekar, S. S.; Pansare, D. N.; Shelke, R. N.; Jawale, C. S.; Shinde, D. B. Bioorg. Med. Chem. Lett. 2016, 26, 3525.

[3] Belveren, S.; Dondas, H. A.; Ulger, M.; Poyraz, S.; GarcíaMingüens, E.; Ferrandiz-Saperas, M.; Sansano, J. M. Tetrahedron 2017, 73, 6718.

[4] Cui, P.-L.; Liu, N.; Chen, H.; Li, X.-L. Chin. J. Org. Chem. 2018, 38, 2784 (in Chinese). (崔朋雷, 刘娜, 陈华, 李小六, 有机化学, 2018, 38, 2784.)

[5] Li, F.-Y.; Guo, X.-F.; Fan, Z.-J.; Zhang, Y.-Q.; Zong, G.-N.; Qian, X.-L.; Ma, L.-Y.; Chen, L.; Zhu, Y.-J.; Tatiana, K.; Morzherin, Y. Y.; Belskaya, N. P. Chin. Chem. Lett. 2015, 26, 1315. 
[6] Zha, G.-F.; Leng, J.; Darshini, N.; Shubhavathi, T.; Vivek, H. K.; Asiri, A. M.; Marwani, H. M.; Rakesh, K. P.; Mallesha, N.; Qin, H.-L. Bioorg. Med. Chem. Lett. 2017, 27, 3148.

[7] Ouf, S. A.; Gomha, S. M.; Ewies, M.; Ouf, A. S.; Sharawy, I. A. Bioorg. Med. Chem. 2018, 26, 3287.

[8] Cao, L.; Sun, J.-W.; Liu, Q.; Qian, C.; Du, Y. L.; Xu, W.-J.; Chen, K.-Y.; Liu, J.-B. Chin. J. Org. Chem. 2017, 37, 3031 (in Chinese). (曹蕾, 孙景伟, 刘强, 钱程, 杜彦林, 许望津, 陈开义, 刘建兵, 有机化学, 2017, 37, 3031.)

[9] Pieroni, M.; Wan, B.; Cho, S.; Franzblau, S. G.; Costantino, G. Eur. J. Med. Chem. 2014, 72, 26.

[10] Abhale, Y. K.; Shinde, A.; Deshmukh, K. K.; Nawale, L.; Sarkar, D.; Mhaske, P. C. Med. Chem. Res. 2017, 26, 2557.

[11] Karale, U. B.; Krishna, V. S.; Krishna, E. V.; Choudhari, A. S.; Shukla, M.; Gaikwad, V. R.; Mahizhaveni, B.; Chopra, S.; Misra, S.; Sarkar, D.; Sriram, D.; Dusthackeer, V. N. A.; Rode, H. B. Eur. J. Med. Chem. 2019, 178, 315.

[12] El-Sabbagh, O. I.; Baraka, M. M.; Ibrahim, S. M.; Pannecouque, C.; Andrei, G.; Snoeck, R.; Balzarini, J.; Rashad, A. A. Eur. J. Med. Chem. 2009, 44, 3746.

[13] Pacca, C. C.; Marques, R. E.; Espindola, J. W. P.; Filho, G. B. O. O.; Leite, A. C. L.; Teixeira, M. M.; Nogueira, M. L. Biomed. Pharmacother. 2017, 87, 381.

[14] Kasralikar, H. M.; Jadhavar, S. C.; Goswami, S. V.; Kaminwar, N. S.; Bhusare, S. R. Bioorg. Chem. 2019, 86, 437.

[15] Rostom, S. A. F.; Faidallah, H. M.; Radwan, M. F.; Badr, M. H. Eur. J. Med. Chem. 2014, 76, 170.

[16] Mirza, S.; Naqvi, A. S.; Khan, K. M.; Salar, U.; Choudhary, M. I. Bioorg. Chem. 2017, 70, 133.

[17] Santana, T. L. D.; Barbosa, M. D. O.; Gomes, P. A. T. D. M.; Cruz, A. C. N. D.; Silva, T. G. D.; Leite, A. C. L. Eur. J. Med. Chem. 2018, 144, 874

[18] Zang, J.; Zhang, J.; Liu, D.; Zhao, L.-X. J. Shenyang Pharm. Univ. 2019, 36, 1074 (in Chinese). (蔵洁, 张健, 刘丹, 赵临襄, 沈阳药科大学学报, 2019, 36, 1074.)

[19] Moldovan, C. M.; Oniga, O.; Parvu, A.; Tiperciuc, B.; Verite, P.; Pîrnau, A.; Crisan, O.; Bojit, M.; Pop, R. Eur. J. Med. Chem. 2011, $46,526$.

[20] Mohareb, R. M.; Zaki, M. Y.; Abbas, N. S. Steroids 2015, 98, 80.

[21] Sinha, S.; Doble, M.; Manju, S. L. Eur. J. Med. Chem. 2018, 158, 34.

[22] Garrepally, P.; Gonugunta, C. S. R. J. Pharm. Res. 2013, 6, 836.

[23] González-Dáaz, H.; Vinã, D.; Santana, L.; Clercq, E. D.; Uriarte, E. Bioorg. Med. Chem. 2006, 14, 1095.

[24] Barcellos, T.; Natavio, M.; Stanczyk, F. Z.; Luo, D.; Jusko, W. J.;
Bender, N. M. Contraception 2019, 100, 283.

[25] Luger, P.; Daneck, K.; Engel, W.; Trummlitz, G.; Wagner, K. Eur. J. Pharm. Sci. 1996, 4, 175 .

[26] Waruiru, R. M. Vet. Parasitol. 1992, 43, 75.

[27] Sellanes, D.; Manta, E.; Serra, G. Tetrahedron Lett. 2007, 48, 1827.

[28] Sellanes, D.; Campot, F.; Núñez, I.; Lin, G.; Espósito, P.; Dematteis, S.; Saldaña, J.; Domínguez, L.; Manta, E.; Serra, G. Tetrahedron 2010, 66, 5384.

[29] Nakamura, Y.; Okumura, K.; Kojima, M.; Takeuchi, S. Tetrahedron Lett. 2006, 47, 239

[30] Zhang, W.; Ma, Z.-H.; Mei, D.; Li, C.-X.; Zhang, X.-L.; Li, Y.-X Tetrahedron 2006, 62, 9966.

[31] Hodgetts, K. J.; Kershaw, M. T. Org. Lett. 2002, 4, 1363.

[32] Priego, J.; Gutierrez, S.;Ferritto, R.; Broughton, H. B. Synlett 2007, 2957.

[33] Beladhria, A.; Beydoun, K.; Ammar, H. B.; Salem, R. B.; Doucet, H. Synthesis 2012, 44, 2264.

[34] Li, Z.-Y.; Zhou, H.-P.; Xu, J.-Y.; Wu, X.-M.; Yao, H.-Q. Tetrahedron 2013, 69, 3281 .

[35] Varma, R. S.; Kumar, D. Org. Lett. 1999, 1, 697.

[36] Thore, S. N.; Gupta, S. V.; Baheti, K. G. Med. Chem. Res. 2013, 22, 3802 .

[37] Ghabbour, H. A.; Kadi, A. A.; ElTahir, K. E. H.; Angawi, R. F.; El-Subbagh, H. I. Med. Chem. Res. 2015, 24, 3194.

[38] Xu, X.; Deng, L.; Nie, L.; Chen, Y.-M.; Liu, Y.-Z.; Xie, R.-R.; Li, Z. Bioorg. Med. Chem. Lett. 2019, 29, 525.

[39] Yadav, A. K.; Srivastava, V. P.; Yadav, D. S. Synth. Commun. 2014, 44, 408

[40] Walczynski, K.; Timmerman, H.; Zuiderveld, O. P.; Zhang, M. Q.; Glinka, R. Il Farmaco. 1999, 54, 533

[41] Cao, X.-T.; Qiao, L.; Zheng, H.; Yang, H.-Y.;Zang, P.-F. RSC Adv 2018, 8,170

[42] Liu, L.; Tan, C.; Fan, R.; Wang, Z.-H.; Du, H.-G.; Xu, K.; Tan, J.-J. Org. Biomol. Chem. 2019, 17, 252.

[43] Kort, M. E.; Atkinson, R. N.; Thomas, J. B.; Drizin, I.; Johnson, M. S.; Secrest, M. A.; Gregg, R. J.; Scanio, M. J. C.; Shi, L.; Hakeem, A. H.; Matulenko, M. A.; Chapman, M. L.; Krambis, M. J.; Liu, D.; Shieh, C. C.; Zhang, X.-F.; Simler, G.; Mikusa, J. P.; Zhong, C. M.; Joshi, S.; Honore, P.; Roeloffs, R.; Werness, S.; Antonio, B.; Marsh, K. C.; Faltynek, C. R.; Krafte, D. S.; Jarvis, M. F.; Marron, B. E. Bioorg. Med. Chem. Lett. 2010, 20, 6812.

[44] Wang, Y.-Y.; Li, Z.-Y.; Huang, Y.; Tang, C.-H.; Wu, X.-M.; Xu, J.-Y.; Yang, H.-Q. Tetrahedron 2011, 67, 7406.

[45] Kumar, S. V.; Parameshwarappa, G.; Ila, H. J. Org. Chem. 2013, 78, 7362.

(Cheng, F.) 